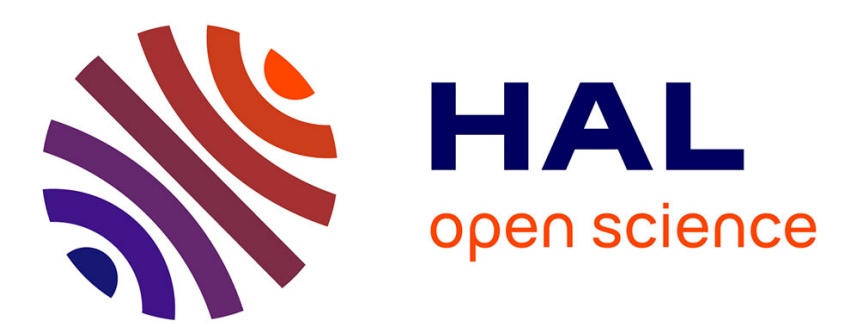

\title{
Naissance des écosystèmes d' affaires : une articulation des compétences intra et inter organisationnelles
}

\author{
Amel Attour, Pierre Barbaroux
}

\section{To cite this version:}

Amel Attour, Pierre Barbaroux. Naissance des écosystèmes d' affaires: une articulation des compétences intra et inter organisationnelles. Gestion 2000, 2016, pp.59-76. hal-03223664

\section{HAL Id: hal-03223664 \\ https://hal.science/hal-03223664}

Submitted on 11 May 2021

HAL is a multi-disciplinary open access archive for the deposit and dissemination of scientific research documents, whether they are published or not. The documents may come from teaching and research institutions in France or abroad, or from public or private research centers.
L'archive ouverte pluridisciplinaire HAL, est destinée au dépôt et à la diffusion de documents scientifiques de niveau recherche, publiés ou non, émanant des établissements d'enseignement et de recherche français ou étrangers, des laboratoires publics ou privés. 


\section{Naissance des écosystèmes d'affaires : une articulation des compétences intra et inter organisationnelles ${ }^{1}$}

Amel Attour (Université Nice-Côte d'Azur, GREDEG)

Pierre Barbaroux (Centre de recherche de l'école de l'air)

Résumé. Cette contribution explore les compétences organisationnelles mobilisées par les acteurs lors de la phase de naissance d'un écosystème d'affaires. La question de recherche posée est la suivante : comment les compétences développées au niveau intra-firme sont articulées au niveau inter-organisationnel lors de la naissance d'un écosystème d'affaires ? Pour traiter cette question, l'article développe une étude de cas portant sur un écosystème de type plateforme. L'étude de cas montre que la phase de naissance peut être décomposée en différences séquences, chacune mobilisant des compétences spécifiques de la part des acteurs. Ensemble, ces compétences permettent d'accomplir les différentes tâches et activités associées à la naissance de l'écosystème d'affaire. Elles forment les composantes de la capacité d'innovation collective déployée par les acteurs lors cette phase critique du cycle de vie de l'écosystème.

Mots clefs. Ecosystèmes d'affaires. Compétences collectives intra firmes. Compétences collectives inter firmes. Cycle de vie.

${ }^{1}$ Article publié dans la revue Gestion 2000, nº , juillet aout, pp. 59-76, 2016. 


\section{Introduction}

Si les écosystèmes d'affaires déployés par les grandes entreprises industrielles et commerciales ont été largement étudiés dans la littérature (Pellegrin-Boucher et Gueguen 2005 ; Ronteau 2009 ; Edouard et Gratacap 2010), la question de la naissance de l'écosystème en tant que forme organisationnelle innovante demeure relativement peu explorée. La plupart des travaux de recherche sur les écosystèmes d'affaires explorent en effet les propriétés de l'architecture relationnelle et industrielle de modèles d'affaires « éco-systémiques » déjà matures ou en phase de croissance et portés par les firmes pivots, leaders de l'écosystème (Adner et Kapoor 2010 ; Koenig 2012; Nambisan et Baron 2013). Ces recherches portent principalement sur les stratégies mises en œuvre par les entreprises pour générer de la valeur au sein d'écosystèmes structurés. Pourtant, la question de l'émergence d'un écosystème d'affaires est importante dans la mesure où nombre de formes organisationnelles innovantes disparaissent avant de croitre, rendant difficile l'étude des déterminants et des capacités déployées lors de la phase de naissance. Parmi les rares travaux qui proposent d'explorer cette question, certains mettent l'accent sur les stratégies des firmes leaders (Mira-Bonnardel et al. 2012); d'autres insistent sur les phases amont du processus d'innovation (Barbaroux 2014a) et de l'échange des connaissances lors de la phase de structuration de l'écosystème naissant (Attour et Ayerbe, 2012, 2015 ; Attour et Della Peruta, 2014 ; Attour et Barbaroux, 2016); d'autres, enfin, explorent le rôle des compétences déployées par les acteurs (Loilier et Malherbe, 2012, 2013).

Quelle que soit la perspective retenue, la phase de naissance d'un écosystème d'affaires relève d'un processus d'apprentissage exploratoire que James Moore qualifie d' «idéation » (Moore 1993, p. 76). Ce processus a pour but premier la mise à l'épreuve d'un concept («proof of concept»; Moore 2006, p. 55) susceptible d'offrir une alternative aux biens et services existants, d'ouvrir un marché nouveau et de générer de la valeur. Les acteurs cherchent alors à 
anticiper un positionnement stratégique sur un marché qui n'est pas encore structuré (technologies immatures, besoins émergents ou latents des clients, environnement réglementaire instable). Durant cette période critique du cycle de vie de l'écosystème, l'enjeu ne se limite toutefois pas à la description de la valeur pour le client réel ou potentiel. Il s'agit d'identifier un espace d'opportunités à travers la recombinaison et/ou la réorganisation des ressources et des compétences des firmes partenaires. Elle engage ainsi le développement de capacités organisationnelles ('value chaining' au sens de Moore, 1996, p.70) permettant de mettre en relation des ressources distribuées pour créer de la valeur.

Dans cette contribution, nous portons l'effort de recherche sur ces compétences mises en œuvre par les acteurs pour faire émerger un écosystème d'affaires nouveau. Si les travaux de Loilier et Malherbe (2012) offrent un cadre d'analyse utile pour identifier les compétences collectivement mobilisées par les acteurs d'un écosystème pendant la phase de naissance, la manière dont les compétences existantes et/ou développées par chaque acteur puis articulées avec celles des autres acteurs n'a cependant pas encore été étudiée. Dans cette optique, la problématique de recherche de l'article est la suivante : comment les compétences développées au niveau intra-firme sont articulées au niveau inter-organisationnel lors de la naissance d'un écosystème d'affaires ? La réponse à cette question est apportée à travers une étude de cas portant sur un écosystème de type plateforme (Gawer, 2014) appelé Sophia Zen.

Le reste de l'article est organisé comme suit. Il commence par définir les différentes séquences de la phase de naissance d'un écosystème d'affaires en identifiant les tâches et les activités réalisées par les différents acteurs de l'écosystème en émergence. Dans cette section, nous suggérons que la réalisation de ces tâches et activités requière la mobilisation de compétences spécifiques. La section suivante présente la méthodologie de l'étude du cas Sophia Zen. Les données collectées ainsi que la façon de les analyser sont alors présentées. La présentation du cas Sophia Zen, section 4, est structurée suivant une grille de lecture qui repose sur les 
différentes séquences de la phase de naissance de l'écosystème. La dernière section enfin, discute les implications et les limites de l'étude de cas et ouvre des perspectives pour la recherche future.

\section{Naissance d'un écosystème d'affaires : la question de l'articulation des compétences des acteurs}

Moore (1993, p. 77) considère que le cycle de vie d'un écosystème d'affaires se compose de quatre phases : naissance, expansion, leadership et régénération (ou déclin). Pour l'auteur, la phase de naissance est elle-même décomposable en quatre séquences (Moore, 1996, p. 109) :

1. La recherche de nouvelles idées.

2. L'action et l'expérimentation.

3. La création de valeur.

4. La rétroaction.

Lors de la première séquence, les acteurs de l'écosystème cherchent à identifier, avant de le construire, un avantage concurrentiel. La recherche de nouvelles opportunités d'affaires est une étape cruciale de la dynamique entrepreneuriale (Shane 2000) sous tendant la naissance d'un écosystème d'affaires. Elle repose sur un processus de découverte (Kirzner 1997) conduit par une firme ou un groupe de firmes dont la finalité est incertaine et qui repose sur des connaissances et des aptitudes spécifiques. La reconnaissance des opportunités d'affaires lors de la première séquence dépend de la capacité des acteurs à donner du sens aux informations disponibles à propos de leur environnement économique, technologique, scientifique et réglementaire (Barbaroux 2014b). Cette capacité cognitive, spécifique de chaque acteur, est conditionnée par ses ressources propres et ses compétences antérieures. Dans la deuxième séquence, les acteurs déterminent une offre primitive et cible une population de clients potentiels. Ces derniers sont sélectionnés en raison de leur ouverture à l'expérimentation : ils 
participent de la conception de la proposition commerciale en partageant leurs expériences d'usage de la future technologie ou du futur service offert par l'écosystème naissant. Les acteurs ajustent ensuite leur proposition de valeur en élargissant leur architecture de valeur au cours de la troisième séquence. De nouveaux membres sont ainsi sollicités pour intégrer l'écosystème afin de disposer des actifs et des processus nécessaires à la mise en œuvre de la proposition de valeur, celle-ci reposant sur une architecture organisationnelle et technologique plus mature. Enfin, lors de la dernière séquence, les acteurs évaluent les actions passées (analyse réflexive) et regardent vers l'avenir pour anticiper les besoins et se préparer aux phases ultérieures du cycle de vie de l'écosystème (expansion et leadership).

Dans la mesure où il n'existe aucune certitude a priori quant à la possibilité de générer de la valeur pour les parties prenantes de l'écosystème naissant, les choix des acteurs vont reposer sur l'ajustement constant des ressources mobilisées, combinées et intégrées. Cette stratégie cognitive entre délibération et programmation d'une part, et improvisation et émergence d'autre part (Mira-Bonnardel et al. 2012, p. 132) est une réponse adaptée aux enjeux soulevés par les différentes séquences qui composent la phase de naissance d'un nouvel écosystème d'affaires. Il s'agit en effet d'une période d'expérimentation et d'apprentissage collectif susceptible de structurer un espace économique, technologique et juridique naissant, propice à la création de valeur et à son appropriation. Cet espace est nécessairement ouvert et collaboratif puisque c'est à travers la mise en relation des acteurs, des ressources et des opportunités d'affaires qu'émergent ces nouvelles formes organisationnelles, industrielles et commerciales.

Cette représentation de la phase amont du cycle de vie de l'écosystème d'affaires est intéressante pour notre propos dans la mesure où elle permet d'identifier les différentes tâches et activités qui doivent être planifiées, organisées et réalisées par les acteurs de l'écosystème naissant. Elle met en avant le rôle crucial des processus d'apprentissage et insiste sur l'importance des relations entre acteurs. Dans ce contexte exploratoire, interactif et collaboratif, 
la naissance d'un écosystème d'affaires peut justifier certaines transformations organisationnelles et opérationnelles, et nécessiter le déploiement de compétences particulières. C'est cette dernière dimension que nous étudions dans cette contribution.

Loilier et Malherbe $(2012$; 2013) ont étudié les compétences éco-systémiques déployés par les acteurs lors de la phase de naissance d'un écosystème. Ainsi, les auteurs identifient trois types de compétences collectives développées tout au long ou lors d'une séquence spécifique de la phase de naissance d'un écosystème de services mobiles (technologie du Near Field Communication ; NFC). Il s'agit des :

- Compétences techniques liées au développement de l'innovation (CTDI),

- Compétences relationnelles favorisant les interactions (CRI),

- Compétences permettant de structurer l'offre (CSO).

Les compétences techniques (CTDI) permettent de développer la technologie et des solutions métier communes (standardisation de la technologie, définition d'interfaces d'interopérabilité technique, pilotage de projets d'expérimentation). Les compétences relationnelles (CRI) relèvent de la mise en relation et de la coordination des acteurs de l'écosystème au niveau des métiers mais aussi au niveau des initiatives prises en commun par ces métiers. Concrètement, il s'agit de mettre en place des associations professionnelles, des consortiums ou des groupes de travail, voire de piloter des projets d'expérimentation. Enfin, les compétences commerciales (CSO) permettent de définir la proposition de valeur de l'écosystème et la répartition des revenus. Elles sont mises en œuvre à travers les modalités suivantes : définition du modèle d'affaires, veille concurrentielle effectuée sur les autres écosystèmes, pilotage de projets d'expérimentation. Dans ce cadre, les auteurs montrent que si les compétences techniques (CTDI) sont développées tout au long des quatre séquences qui structurent la naissance de l'écosystème étudié, les compétences relationnelles (CRI) sont développées pendant la 
séquence «création de valeur» tout comme les compétences commerciales (CSO) dont le développement se poursuit durant la rétroaction.

La typologie proposée par Loilier et Malherbe $(2012$; 2013) offre une grille de lecture pertinente si l'on cherche à comprendre la nature et la diversité des compétences interorganisationnelles sous-tendant la naissance d'un écosystème d'affaires de type plateforme, ainsi que la chronologie de leur développement. La proposition des auteurs souffre toutefois d'un écueil majeur. Les auteurs précisent en effet que leur « niveau d'analyse du développement des compétences est (...) inter organisationnel et s'inscrit dans la lignée des travaux sur les « compétences collaboratives (...) et inter organisationnelles» (Loilier et Malherbe 2012, p. 91). Or, rien n'est dit sur les relations entre les compétences éco-systémiques et les compétences et les ressources internes des acteurs. Quels liens les compétences collectives (techniques, relationnelles et commerciales) entretiennent-elles avec les compétences internes des membres de l'écosystème? Ces dernières ont-elles un effet habilitant ou contraignant sur le développement des compétences collectives? Il apparaît que les auteurs n'étudient pas directement l'articulation des compétences internes des membres de l'écosystème naissant avec les compétences co-construites lors des différentes séquences de la phase de naissance. Or, cette question est essentielle. L'innovation collaborative sur laquelle repose la structuration de l'écosystème naissant suppose de mettre en relation, à travers des mécanismes de combinaison, d'intégration et d'alignement, une variété de ressources et de compétences à la fois spécifiques et distribuées (Barbaroux 2012, p. 234-235). Le déploiement d'une capacité d'innovation collaborative suppose la mobilisation et la combinaison par les acteurs de ressources internes qui apparaissent (i) favorables à l'échange (e.g., capacités d'absorption et de transfert des connaissances tacites ; Cohen et Levinthal 1990), (ii) complémentaires sur le plan technique (e.g., interopérabilité des plateformes et des services ; Love et Roper 2009) et (iii) consonantes sur le plan culturel (e.g., valeurs et normes, confiance mutuelle, engagement réciproque ; 
Lawrence et Scanlan 2007 ; Miles 2007). Dans cette optique, il convient d'étudier comment les compétences internes apportées par les acteurs s'articulent avec les compétences écosystémiques identifiées par Loilier et Malherbe (2012) pour former une capacité d'innovation collaborative spécifique de l'écosystème naissant, L'étude de cas développée dans cette contribution a justement pour but de traiter cette question.

\section{Méthodologie}

La méthodologie mobilisée est de nature qualitative. Elle s'inscrit dans une démarche exploratoire et privilégie une approche basée sur une étude d'un cas unique (Stake, 1995) portant sur le projet Sophia-Zen, un écosystème-plateforme (Gawer 2014) du secteur des technologies de type Near Field Communication (NFC). L'étude d'un cas unique repose sur une démarche d'analyse exploratoire (Yin, 1989). La méthode de recherche retenue s'inscrit dans une démarche inductive de collecte de données par l'observation in situ, pour observer dans son contexte naturel un phénomène mal connu (David, 2000). Il s'agit plus précisément d'une étude de cas de type contemplative (Savall et Zardet, 2004). Ainsi, les données primaires ont pour première source :

- La participation aux différentes réunions de montage du projet Sophia-Zen (menées en présentiel, par téléphone ou au cours de visioconférences) par l'un des auteurs.

- La participation mais aussi les comptes rendus de réunions auxquelles l'un des auteurs de l'article a participé dans les différentes phases du projet.

Cette première source de données primaires (comptabilisant au total 10 réunions et 2 séances de brainstorming) a été complétée par des données secondaires issues des documents techniques mis à la disposition du chercheur par les praticiens. Il s'agit des rapports d'activités du projet, de la collecte d'informations disponibles en ligne sur des projets d'expérimentation similaires auxquels un ou plusieurs acteurs du cas étudié ont également eu l'occasion de participer. 
L'analyse thématique inductive de ces informations (Paillé, 1996) nous a conduit à identifier, à partir du contenu des données primaires, quatre catégories de compétences considérées comme des aptitudes à réaliser certaines tâches essentielles lors des différentes séquences de la phase de naissance. L'association des aptitudes et des séquences nous a ensuite permis d'organiser la présentation des résultats.

\section{Présentation du cas Sophia-Zen}

Le projet Sophia-Zen a regroupé plusieurs acteurs: la société de services en ingénierie informatique (SSII) GFI Informatique ${ }^{2}$ (GFI), l'Université de Nice Sophia-Antipolis (UNS), le réseau de transports en commune de Sophia Antipolis (Envibus) et la Communauté d'Agglomération de Sophia-Antipolis (CASA). Financé par le dispositif PacaLabs du Conseil Régional Provence-Alpes-Côte-D’azur (PACA), le projet Sophia Zen a débuté en septembre 2011 et s'est achevé septembre 2012, Il a eu pour finalité de déployer un service de covoiturage dynamique couplant deux plateformes technologiques qui constituent les deux composantes de Sophia-Zen : la composante CHEMIN (Chemins de l'Histoire E-guidés par Mobiles Intégrants le NFC) déployée par l'UNS et la composante ENTREPRISE déployée par GFI.

La première composante, CHEMIN, propose un service destiné aux personnes en situation de mobilité sur la technopole. Elle est constituée d'un service d'informations multimédia (vidéos, audio, photos) reçus ou produits sur le téléphone mobile personnel de l'utilisateur final et d'un service de géolocalisation. Cette fonctionnalité de base offre est destinée à des utilisateurs en situation de mobilité munies d'un téléphone mobile NFC (ou compatible NFC) la possibilité de suivre un chemin prédéfini et d'avoir ainsi une description des lieux (vidéos, audio, photos, commentaires associés à une borne). En pratique, CHEMIN permet aux utilisateurs de faire une

\footnotetext{
${ }^{2}$ Acteur européen de référence des services informatiques à valeur ajoutée et des logiciels.
} 
visite guidée, grâce à un système de bornes dotées de tags NFC positionnées à des endroits importants d'un territoire.

La deuxième composante de Sophia-Zen, ENTREPRISE, a pour point de départ l'application gratuite pour iPhone «TPG » (Transports Publics Genevois) qui indique, en temps réel, les prochains passages des bus de la ville de Genève grâce à leur position GPS exacte. L'objectif de GFI est de rendre cette solution multimodale en lui associant un système de géolocalisation identique au système de localisation mondial (le Global Positioning System - GPS) mais spécifique au territoire où ce système est déployé. Il vise en particulier à résorber les inconvénients liés au GPS 'standard' qui peut ne pas donner la position exacte ou ne pas connaître le lieu recherché. En plus de pallier ce manque de précision, la composante ENTREPRISE est une solution mettant à jour les positions et descriptions des entreprises de la technopole (en cas de déménagement d'une entreprise sur le site de Sophia-Antipolis par exemple).

Dans la suite de cette partie, la présentation de l'étude de cas est structurée sur la base des activités et des tâches réalisées par les acteurs au cours des quatre séquences de la naissance de l'écosystème Sophia-Zen.

\subsection{Idéation : la formulation du concept Sophia-Zen}

La séquence d'idéation de Sophia-Zen est antérieure à la date de début du projet. Elle débute en juillet 2010, lorsque le responsable scientifique du Master MBDS de l'UNS convie ses trois chefs de projets à une séance de brainstorming dont l'objectif est d'identifier comment répondre à un appel à projets (PacaLabs) publié par la Région Provence-Alpes-Côte-D'azur (PACA). De cette séance de brainstorming émerge l'idée de saisir l'opportunité du PacaLabs pour unifier les versions antérieures de CHEMIN et l'enrichir d'un service de géolocalisation permettant de résoudre les problèmes de congestion existants sur la technopole de Sophia-Antipolis. 
CHEMIN a en effet connu trois versions antérieures respectivement déployées dans les villes de Nice, Menton et Grasse. Chacune de ces trois versions avait été mise en œuvre sous la supervision d'un chef de projet différent. De fait, il devenait essentiel de capitaliser le retour des expérimentations menées par chacun pour ne disposer que d'une seule version. Cette idée a ensuite été défendue par les chefs de projets auprès du responsable scientifique en mettant l'accent sur la nécessité de disposer d'une version unique de la composante CHEMIN, de corriger les problèmes existants dans les versions antérieures (e.g. la non-gestion des relations entre les différentes entités impliquées dans la plateforme qui provoquait de nombreux plantages du site web) et de faire évoluer les fonctionnalités existantes avec les nouvelles technologies (e.g. passer de la version 1 à 3 l'API ${ }^{3}$ Googlemap $^{4}$, intégrer les applications mobiles de type Android, etc.). Une fois cette idée validée en interne, avec l'aide de ses trois chefs de projet, le responsable scientifique s'est attaché à identifier et à solliciter l'acteur externe qui complétera l'offre CHEMIN. La décision finale a porté sur GFI. Cet acteur disposait de l'application TPG qu'il souhaitait expérimenter et déployer en France.

\subsection{Action et expérimentation : le choix des options technologiques}

Septembre 2011 marque le début de la séquence « action et expérimentation ». Cette deuxième séquence, les premiers développements techniques ont d'abord été au niveau intra firme (au sein des équipes de chaque acteur de Sophia-Zen), puis au niveau inter-firme (entre les deux acteurs de Sophia-Zen). Pour l'UNS, il s'agissait de fusionner les différentes versions existantes de la plateforme CHEMIN en une unique version et de faire évoluer certaines composantes (soit pour résoudre des problèmes techniques existants, soit pour innover). Pour GFI, l'enjeu a

\footnotetext{
${ }^{3}$ API est un acronyme pour Applications Programming Interface. Une API est une interface de programmation qui permet de se "brancher" sur une application pour échanger des données.

${ }^{4}$ L'API de Googmap permet de localiser tout type de données sur une carte (routière, satellite, mixte) à partir de son adresse postale. Cet api s'avère très utile pour proposer aux internautes une vision globale et géographique de données (membre d'une communauté, restaurants d'un quartier...).
} 
surtout consisté à rendre interopérable la composante existante «TPG ». Le tableau 1 présente les composantes de CHEMIN et d'ENTREPRISE qui ont fait l'objet de modifications.

\begin{tabular}{|c|c|}
\hline Composantes de CHEMIN & Illustration \\
\hline \multicolumn{2}{|r|}{ UNS } \\
\hline $\begin{array}{l}\text { Architecture du site web } \\
\text { administratif }\end{array}$ & $\begin{array}{l}\text { Création des URLs des parcours, évolution de l'API } \\
\text { Googlemap de la version } 1 \text { à } 3 \text {, modification de l'upload } \\
\text { des médias, etc. }\end{array}$ \\
\hline $\begin{array}{l}\text { Evolution du format de collecte } \\
\text { des statistiques }\end{array}$ & $\begin{array}{l}\text { Désormais basé sur la fréquentation des différents médias } \\
\text { par les visiteurs depuis leur téléphone mobile (application } \\
\text { Android) et non basées sur le nombre de création par jour) } \\
\text { a nécessité la création des entités, des sessions Bean et } \\
\text { des web services. }\end{array}$ \\
\hline $\begin{array}{l}\text { Processus de création et de } \\
\text { gestion des bornes de tag NFC }\end{array}$ & $\begin{array}{l}\text { Une borne n'est dorénavant associée qu'à un seul tag } \\
\text { (« visite »); l'utilisateur ne peut plus accéder directement } \\
\text { aux médias et commentaires en scannant un tag du fait } \\
\text { qu'une borne peut être le point de passage de plusieurs } \\
\text { parcours }\end{array}$ \\
\hline \multicolumn{2}{|r|}{ GFI } \\
\hline $\begin{array}{l}\text { Rendre interopérable } \\
\text { l'application « TPG » }\end{array}$ & $\begin{array}{l}\text { Evolution de l'application «TPG » vers un système } \\
\text { Android }\end{array}$ \\
\hline
\end{tabular}

Tableau 1. Evolution des composantes de CHEMIN et ENTREPRISE

$\mathrm{Au}$ niveau inter-firme ensuite, les développements techniques ont essentiellement porté sur la manière dont les deux composantes peuvent être interconnectées de manière à proposer à l'utilisateur final une offre globale. 


\subsection{Création de valeur : l'identification des utilisateurs}

Pendant la troisième séquence, les acteurs de Sophia-Zen élaborent le modèle économique de l'offre globale en dissociant les modèles de revenus propres à chaque composante de la plateforme. Il a donc été nécessaire de distinguer l'utilisateur final de la cible finale d'une part, le potentiel de valeur pour le client d'autre part.

Pour CHEMIN, l'utilisateur final identifié est un individu ou un groupe d'individus en situation de mobilité (touristes, touristes d'affaires, etc.) mais le client de la plateforme ne peut qu'être un acteur territorial (dans le cas Sophia-Zen cet acteur est la CASA). En effet, la composante CHEMIN est perçue par la communauté d'agglomération comme une solution permettant de dynamiser le territoire sophipolitain, notamment sur le plan culturel. La première offre de service de CHEMIN est ainsi de guider les personnes en situation de mobilité sur un chemin culturel ou historique, et de proposer différentes options de traverse et de promenade réelles dans des espaces 'virtuels'. Il est également apparu que la composante pouvait offrir une solution permettant de faciliter la circulation sur le territoire. Ainsi, un service de consultation en temps réels des horaires des transports en commun a été proposé aux utilisateurs finaux. Cet enrichissement de l'offre a nécessité des développements technologiques supplémentaires en intégrant certaines contraintes d'usage (ergonomie de l'interface, facilité d'accès et d'affichage des informations, personnalisation).

La composante ENTREPRISE vise la même catégorie d'utilisateurs finaux que CHEMIN. Toutefois, le client change de nature puisqu'il s'agit des entreprises implantées sur le territoire de la communauté d'agglomération de Sophia-Antipolis. En effet, celles-ci souffrent d'un problème de géolocalisation de la part de leurs clients, fournisseurs et partenaires dû au déficit de signalisation existant sur le territoire. Le concept est donc de permettre aux employés, partenaires des entreprises localisées dans la technopole mais aussi aux touristes d'affaires de s'orienter et de s'informer en temps réel sur une zone d'activité ou d'entreprises. Ainsi l'offre 
de services de la composante ENTREPRISE a été enrichie de deux applications supplémentaires. Un plan embarqué pour s'orienter (la solution « Je suis perdu ») et une solution de covoiturage dynamique (la solution «Je me déplace ») qui permet aux employés (mais aussi aux étudiants) de communiquer entre eux en temps réel et de faciliter leurs déplacements (fiche horaires des bus, parcours emprunté, info trafic, etc.). Ensemble, les solutions de services, les utilisateurs et les clients potentiels associés à chacune des composantes de la plateforme constituent les fondements de la proposition de valeur du modèle économique Sophia-Zen.

\subsection{Rétroaction : vers une plateforme intégrée?}

La rétroaction dans le cas Sophia-Zen est une phase réflexive quant à la stratégie commerciale adoptée par les acteurs. Durant cette ultime étape de la naissance de Sophia-Zen, les acteurs font le choix de commercialiser séparément les deux composantes en partie en raison de la cible de clients qui diffère pour les deux composantes CHEMIN et ENTREPRISE. UNS a ainsi développé un modèle d'appropriation «ouvert» dans lequel les clients de la composante CHEMIN peuvent accéder au code source et adapter les applications proposées aux utilisateurs finaux. GFI, en revanche, a opté pour un modèle propriétaire «fermé » dans lequel les clients de la composante ENTREPRISE n'ont pas le droit de modifier le contenu et les applications offertes.

\section{Naissance de Sophia-Zen : mobilisation des compétences intra et inter}

\section{firmes}

L'analyse des données recueillies suggère, à la lumière des travaux de Loilier et Malherbe (2012), que l'UNS et GFI mobilisent plusieurs compétences dans les différentes séquences qui structurent la naissance de Sophia-Zen (Tableau 2). Cependant, nos résultats montrent que la mobilisation de ces compétences suit une logique différente de celle décrite par Loilier et Malherbe (2012). cette divergence est observée à deux niveaux. Au niveau chronologique 
d'abord, puis à l'échelle organisationnelle. La lecture du tableau 2 montre que seules les compétences commerciales de structuration de l'offre (CSO) sont mobilisées au cours des mêmes séquences identifiées par Loilier et Malherbe (2012), i.e. les séquences 3 et 4 de la naissance de Sophia Zen. En revanche, lorsque les auteurs affirment que les compétences techniques sont mobilisées tout au long des quatre séquences qui structurent la naissance de l'écosystème étudié, nous montrons que pendant la séquence rétroaction de Sophia Zen les acteurs font uniquement appel à des compétences commerciales (CSO) et relationnelles (CRI). Enfin, les compétences relationnelles sont dans le cas de Sophia Zen mobilisées tout au long de la naissance de l'écosystème, alors que pour Loilier et Malherbe (2012) elles interviennent uniquement durant la séquence « création de valeur ».

Ces divergences viennent en partie du niveau d'analyse choisis par les auteurs. L'identification des compétences collectives est centrée sur le niveau inter-organisationnel et ne considère pas l'articulation des compétences internes des membres de l'écosystème naissant avec les compétences co-construites. Or, comme le montre le cas Sophia-Zen cette articulation est essentielle à la naissance de l'écosystème.

\subsection{Naissance de Sophia Zen : des compétences intra aux compétences inter firmes}

Comme le souligne la lecture du tableau 2, la séquence d'idéation fait intervenir un seul acteur de l'écosystème: l'UNS. Par conséquent, les compétences collectives mobilisées sont uniquement de type intra firmes. Il s'agit de compétences techniques (CTDI) et commerciales (CSO) mobilisées par les chefs de projet de l'UNS pour identifier le concept de Sophia-Zen. La mobilisation des compétences techniques a permis de faire émerger l'architecture technique de CHEMIN incluant non seulement l'interface web de la plateforme mais également une exploration des situations d'usages potentielles (gestions des bornes NFC, gestion des parcours, gestion des médias, gestion des commentaires, etc.). Les différentes interactions entre les chefs de projets, par le dialogue lors de séances de brainstorming, ont également permis de structurer 
l'offre primitive du projet et d'identifier l'endossement de CHEMIN à un service de géolocalisation territorialisé comme principale source de création de valeur. En outre, cette première séquence a été déclenchée par l'aptitude du responsable scientifique de l'UNS à réunir et coordonner ses trois chefs de projets de l'UNS (notamment à travers l'organisation de différentes séances de brainstorming). Ce résultat suggère que, si au niveau inter-firme les compétences relationnelles (CRI) sont mobilisées et développées à partir de la séquence «création de valeur » (Loilier et Malherbe, 2012), ces dernières le sont au niveau intra-firme, dès la séquence idéation.

Dans le cas spécifique de Sophia-Zen, le développement de l'architecture technique permettant de connecter les deux composantes CHEMIN et ENTREPRISE a non seulement mobilisé des compétences techniques propres à chaque acteur mais aussi des compétences relationnelles conjointement développés entre les chefs de projet du MBDS et les chefs de projets GFI. De manière plus générale, l'observation des séquences «action et expérimentation » et « création de valeur » révèle, comme le décrivent par ailleurs les sections 4.2. et 4.3. , que les activités de développement technologique et de définition de la proposition de valeur du modèle d'affaires de Sophia-Zen sont d'abord menées au sein de chaque composante de l'écosystème. De fait, les compétences techniques et commerciales propres à chaque acteur de l'écosystème ont été mobilisées au niveau intra firme. Ces compétences ont permis pour chaque composante de Sophia-Zen d'identifier les services, développés au niveau intra-firme, permettant d'enrichir l'offre des deux composantes. La coordination des activités menées individuellement par les deux acteurs permet ensuite de formuler un potentiel d'innovation collectif. Cette coordination mobilise des compétences relationnelles inter firmes mises en œuvre par les responsables (scientifiques) respectifs de CHEMIN et ENTREPRISE. Il s'avère alors essentiel de comprendre comment cette intégration des connaissances externes influence le développement de l'innovation ainsi que les choix relatifs à la structuration de l'offre au niveau inter-firmes. 


\begin{tabular}{|c|c|c|c|c|}
\hline \multirow[t]{2}{*}{ Séquence } & \multicolumn{2}{|c|}{$\begin{array}{c}\text { Activités réalisées au niveau } \\
\text { intra-firme }\end{array}$} & \multicolumn{2}{|c|}{$\begin{array}{c}\text { Activités réalisées au niveau } \\
\text { inter-firmes }\end{array}$} \\
\hline & Activités & $\begin{array}{c}\text { Compétences } \\
\text { mobilisées }\end{array}$ & Activités & $\begin{array}{c}\text { Compétences } \\
\text { mobilisées }\end{array}$ \\
\hline Idéation & $\begin{array}{l}\text { Formulation du } \\
\text { concept de } \\
\text { Sophia-Zen : } \\
\text { coupler la version } \\
\text { unifiée de } \\
\text { CHEMIN avec } \\
\text { ENTREPRISE } \\
\text { Choix de l'acteur } \\
\text { externe à } \\
\text { CHEMIN }\end{array}$ & $\begin{array}{c}\text { Techniques } \\
\text { et } \\
\text { Commerciales } \\
\text { Relationnelles }\end{array}$ & & \\
\hline $\begin{array}{l}\text { Action et } \\
\text { expérimentation }\end{array}$ & $\begin{array}{l}\text { UNS : } \\
\text { Unification des } 3 \\
\text { versions } \\
\text { antérieures de } \\
\text { CHEMIN et } \\
\text { amélioration de } \\
\text { certaines } \\
\text { composantes de } \\
\text { CHEMIN } \\
\text { GFI : } \\
\text { développement } \\
\text { interopérabilité } \\
\text { de TPG }\end{array}$ & Techniques & $\begin{array}{c}\text { UNS et GFI : } \\
\text { développement de } \\
\text { l'architecture } \\
\text { technique } \\
\text { permettant de } \\
\text { connecter les } \\
\text { deux composantes } \\
\text { CHEMIN et } \\
\text { ENTREPRISE }\end{array}$ & $\begin{array}{c}\text { Relationnelles } \\
\text { Techniques }\end{array}$ \\
\hline $\begin{array}{l}\text { Création de } \\
\text { valeur }\end{array}$ & $\begin{array}{l}\text { Distinction entre } \\
\text { l'utilisateur final } \\
\text { et la cible finale } \\
\text { pour chaque } \\
\text { composante } \\
\text { CHEMIN et } \\
\text { ENTREPRISE }\end{array}$ & Techniques & $\begin{array}{l}\text { Validation du } \\
\text { concept final de } \\
\text { Sophia-Zen }\end{array}$ & Relationnelles \\
\hline Rétroaction & $\begin{array}{l}\text { Evaluation des } \\
\text { modèles de } \\
\text { revenus élaborés } \\
\text { en séquence } 3\end{array}$ & $\begin{array}{l}\text { Commerciales } \\
\text { Relationnelles }\end{array}$ & $\begin{array}{c}\text { choix de séparer } \\
\text { la } \\
\text { commercialisation } \\
\text { des deux } \\
\text { composantes }\end{array}$ & $\begin{array}{l}\text { Commerciales } \\
\text { Relationnelles }\end{array}$ \\
\hline
\end{tabular}

Tableau 2. Les compétences éco-systémiques mobilisées pendant la phase naissance de Sophia-Zen

5.2. Naissance de Sophia Zen : une articulation des compétences intra et inter firmes 
Le cas Sophia-Zen révèle que le rôle des compétences relationnelles est prédominant sur le plan inter-organisationnel, alors que les compétences techniques et commerciales s'avèrent davantage mobilisées au niveau intra-firme. Il s'avère en effet que les compétences relationnelles ont pour principal rôle l'articulation des développements réalisés à l'appui des compétences intra firmes de types techniques et commerciales. Nos résultats suggèrent en effet que dans un environnement collaboratif l'intégration des connaissances externes reposent sur le déploiement de dispositifs facilitant l'interaction entre les acteurs. Dans le cas spécifique de Sophia Zen ces dispositifs prennent la forme de techniques d'idéation (séances de brainstorming par exemple).

Outre le rôle des compétences relationnelles intra firmes mobilisées et ensuite développées au niveau inter-firmes, notre recherche permet d'identifier quelle(s) capacité(s) facilitent l'intégration, et plus généralement, l'articulation des compétences développées au niveau intrafirme. Il apparaît ainsi que l'aptitude des acteurs à absorber des connaissances (Cohen et Levinthal 1990) joue un rôle déterminant. Initialement dépendante des connaissances antérieures accumulées par les acteurs de l'écosystème (Noblet et Simon, 2010), la formulation d'une proposition de valeur a ainsi reposé sur l'aptitude des acteurs à reconnaître, intégrer et valoriser des informations externes en les combinant avec leurs ressources internes (Zahra et George, 2002).

Nos résultats indiquent plus largement que l'aptitude des acteurs à mettre en cohérence leurs options technologiques avec les besoins d'utilisateurs/clients d'une part, et avec leurs stratégies et leurs représentations d'autre part, est décisive notamment lorsqu'il s'agit de formaliser la proposition de valeur. Cette compétence contribue en effet à la formulation d'une offre primitive (au sens de Moore 1996) dont nos résultats suggèrent que la réalisation va dépendre de l'aptitude des acteurs à ajuster leur proposition de valeur et à en maintenir la cohérence. 
Le cas Sophia-Zen montre enfin que le choix des acteurs de rompre avec la stratégie collaborative initiale et de mettre en œuvre leur propre modèle d'appropriation, a suscité une rupture de la proposition de valeur. Celle-ci trouve sa source, non pas dans l'immaturité technologique de la plateforme ou dans l'inadéquation des services offerts au regard des besoins des utilisateurs, mais dans la divergence des stratégies de valorisation des acteurs. Ce constat a pour conséquence de souligner l'importance d'anticiper les enjeux d'appropriation de la valeur dès les séquences amont de la phase de naissance de l'écosystème. De plus, contrairement à la position défendue par Moore (1996), l'aptitude des acteurs à ajuster continûment leur proposition de valeur ne garantit pas nécessairement la naissance, et donc l'expansion, de l'écosystème en tant que forme organisationnelle collaborative. Dans le cas Sophia-Zen, la dynamique réflexive collective s'est traduite par l'abandon du projet collaboratif initial au profit du développement parallèle de deux plateformes distinctes. Sur le plan technologique, les acteurs du projet Sophia-Zen ont ainsi été capables d'élaborer une proposition de valeur répondant à des besoins individuels, institutionnels et économiques. Cependant, la nécessité d'intégrer, au sein d'une même plateforme, deux composantes distinctes s'est traduite par l'impossibilité d'adopter un modèle économique unique. Les acteurs ont par conséquent choisi d'exploiter commercialement chacune des deux composantes de façon séparée, donnant alors naissance à deux écosystèmes-plateformes distincts.

\section{Conclusion}

L'objectif de cette contribution était d'offrir un cadre d'analyse de la phase de naissance d'un écosystème d'affaires qui repose sur l'identification et l'articulation des compétences mises en œuvre par les firmes. En dépit d'avancées significatives de la recherche, nous n'avons en effet qu'une connaissance parcellaire de la spécificité des compétences sous-tendant l'émergence des formes interactives et collaboratives d'organisation du processus d'innovation. Cette grille de lecture proposée doit être considérée comme le résultat d'une recherche exploratoire. Des 
recherches supplémentaires sont évidemment nécessaires en vue d'évaluer la validité empirique ainsi que la pertinence théorique de cette proposition. Une voie de recherche intéressante consisterait à étudier les compétences supportant le développement des formes organisationnelles différentes des écosystèmes d'affaires, comme les modèles d'innovation communautaire (l'innovation comme communauté de communautés), ouverte (l'open innovation) ou participative (crowdsouring).

\section{Bibliographie}

Adner, R., Kapoor, R., 2010, «Value creation in innovation ecosystems: How the structure of technological interdependence affects firm performance in new technology generations», Strategic Management Journal, vol. 31, p. 306-333.

Attour, A., Ayerbe, C. (2012), «Connaissances et innovation au sein des écosystèmes d'affaires. Le cas des services mobiles », Revue Française de Gestion, n²21, p. 77-94.

Attour, A., Ayerbe, C. (2015), «Le management amont et aval des droits de propriété intellectuelle au sein des écosystèmes-plateformes naissants » (avec C. Ayerbe), Système d'Information et Management, $\mathrm{n}^{\circ} 3$, vol.20,2015, p.47-96.

Attour, A., Barbaroux, P., 2016, «Architectural knowledge and the birth of a platform ecosystem», Innovations. Journal of Innovation Economics \& Management, à paraître $\left(1^{\text {er }}\right.$ trimestre 2016).

Attour, A., Della Peruta M., 2014, «Architectural Knowledge: key flows and processes in designing an inter-organizational technological platform », Knowledge Management Research \& Practice, advance online publication 4 August 2014; doi: 10.1057/kmrp.2014.21.

Barbaroux, P., 2012, "Identifying collaborative innovation capabilities: Insights from the ARPANET project”, European Journal of Innovation Management, vol. 15, n², p. 232-258. 
Barbaroux, P., 2014a, «Rupture technologique et naissance d'un écosystème : Voyage aux origines de l'Internet», Revue d'Economie Industrielle, 146/2, p. 27-59.

Barbaroux, P., 2014b, "From market failures to market opportunities: Managing innovation under asymetric information”, Journal of Innovation and Entrepreneurship, vol. 3, $\mathrm{n}^{\circ} 15,15$ pages.

Barbaroux, P., Attour, A., 2016, «Approches interactives de l'innovation et gestion des connaissances », Innovations. Revue d'Economie et de Management de l'Innovation, à paraître ( $1^{\mathrm{er}}$ trimestre 2016).

Burgelman, R.A., Maidique, M., Wheelright, S., 2004, Strategic Management of Technology and Innovation, McGraw-Hill, New York.

Cohen, W., Levinthal, D. 1990, «Absorptive capacity: A new perspective on learning and innovation», Administrative Science Quarterly, 35, p. 128-152.

David, A., 2000, «Logique, épistémologie et méthodologie en sciences de gestion : trois hypothèses revisitées», in Les nouvelles fondations des sciences de gestion - Eléments d'épistémologie de la recherche en management, A. David, A. Hatchuel, R. Laufer (eds.), Vuibert, Paris, p. 83-110.

Edouard, S., Gratacap, A., 2010, «Configuration des écosystèmes d'affaires de Boeing et d'Airbus : le rôle des TIC en environnement innovant », Management \& Avenir, vol. 34, $\mathrm{n}^{\circ} 4$, p. $162-182$.

Gawer, A., 2014, «Bridging differing perspectives on technological platforms: Toward an integrative framework», Research Policy, 43, p. 1239-1249.

Girod, M., 1995, Mémoire et organisation, Thèse de troisième cycle, Université Paris Dauphine, juin 1995. 
Kirzner, I., 1997, "Entrepreneurial discovery and the competitive market process: An Austrian approach”, Journal of Economic Perspectives, vol. 35, n¹, p. 60-85.

Koenig, G., (2012), «Le concept d'écosystème d'affaires revisité», M@n@gement, vol. 15, n 2, p. 209-224.

Lawrence, P., Scanlan, J. 2007, "Planning in the dark: why major engineering projects fail to achieve key goals", Technology Aanalysis \& Strategic Management, vol. 19, n4, p. 509-525. Loilier, T., Malherbe, M., 2012, «Le développement des compétences éco systémiques. Le cas de l'ESA émergent des services mobiles sans contact», Revue Française de Gestion, n 222 , 2012/3, p.89-105.

Loilier, T., Malherbe, M. 2013, «Experimentation and the development of eco-systemic competencies in the field of contactless mobile services», in Understanding business ecosystems, S. Ben Letaifa (ed.), De Boeck, Paris, p.193-207.

Lopez-Nicolas, C., Merono-Cerdan, A.L., 2011, «Strategic knowledge management, innovation and performance», International Journal of Information Management, 31, 502-509. Love, J.H., Roper, S., 2009, “Organizing the innnovation process: complementarities in innovation networking", Industry and Innovation, vol. 16, p. 273-290.

Miles, R.E., 2007, "Innovation and leadership values”, California Management Review, Vol. $50, \mathrm{n}^{\circ} 1$, p. $192-201$

Mira-Bonnardel, S., Geniaux, I., Serrafero, P., 2012, « Naissance d'un écosystème d'affaires : entre stratégie chemin délibérée et stratégie chemin faisant », Revue Française de Gestion, $\mathrm{N}^{\circ}$ $222,2012 / 3$, p. 123-134.

Moore, J., 1993, «Predators and Prey: a new ecology of competition», Harvard Business Review, Vol.71, n³, p.75-86. 
Moore, J., 1996, « The Death of Competition - Leadership and Strategy in the Age of Business Ecosystems», Harper Business.

Moore, J. 2006, «Business ecosystems and the view from the firm», The Antitrust Bulletin, Vol. 51, $\mathrm{N}^{\circ} 1$, p. 31-75.

Nambisan, S., Baron, R.A., 2013, « Entrepreneurship in innovation ecosystems: Entrepreneurs’ self-regulatory processes and their implications for new venture success», Entrepreneurship Theory \& Practice, 37(5), p. 1071-1097.

Noblet, J.P., Simon, E., 2010, « Capacité d'absorption : revue de littérature, opérationnalisation et exploration», Gestion 2000, vol.27, n6, p.59-74.

Paillé, P., 1996, De l'analyse qualitative en général et de l'analyse thématique en particulier, vol.15 : Recherches Qualitatives, p.179-194.

Pellegrin-Boucher, E., Gueguen, G., 2005, «Stratégies de «coopétition » au sein d'un écosystème d'affaires : une illustration à travers le cas de SAP », Finance, Contrôle, Stratégie, vol. $8, \mathrm{n}^{\circ} 1$, p. 109-130.

Ronteau, S., 2009, «Embrasser la condition de firme-pivot : dynamiques d'innovation de Dassault Systèmes dans son écosystème d'affaires », Management \& Avenir, 2009/8, n 28, p. $196-215$.

Savall, H., Zardet, V. 2004, Recherche en sciences de gestion : approche qualimétrique Observer l'objet complexe, Economica.

Shane, S., 2000, "Prior knowledge and the discovery of entrepreneurial opportunities", Organization Science, Vol. 11, n²4, p. 448-469.

Stake, R., 1995, The art of case study research, Thousand Oaks, London, New Delhi: Sage. 
Wang, C.L., Ahmed, P.K., 2007, «Dynamic capabilities: A review and research agenda», International Journal of Management Reviews, vol.9, $\mathrm{n}^{\circ} 1, \mathrm{p} .31-51$.

Yin, R.L., 1989, Application of Case Study Research, Applied Social Research Series, Second Edition, 34, Sage Publications.

Zahra, S.A., George, G., 2002, «Absorptive capacity: a review, reconceptualization, and extension», Academy of Management Review, 27(2), p.185-203. 